\title{
Towards a comprehensive diagnostic assay for scoliosis
}

Adolescent idiopathic scoliosis (AIS) is one of the most common childhood deformities worldwide, characterized by a 3D spinal deformity with unknown cause, and represents both an immediate medical challenge and a chronic condition affecting individuals throughout their lives. The standard of care for scoliosis has not changed in any significant manner in decades. Patients today are treated in a substantially similar manner to those 20 or 30 years ago: observation, bracing and spinal surgery as last resort. Recent progress allow the identification of potential candidate genes, but the function of these still remains elusive and further efforts should be made to connect the predisposing genetic background to the physiopathology. To overcome that situation, we developed functional and biochemical assays that represent promising alternatives. They can help to understand the physiopathology of AIS and direct genetic studies, but more importantly they will contribute to an improved stratification of AIS patients, and thus lead to accurate personalized diagnoses, prognoses and treatment strategies.

\section{KEYWORDS: biochemical markers cellular dielectric spectroscopy genetics genomics Gi-protein signaling idiopathic scoliosis pediatric population personalized medicine whole-cell assay}

Spinal deformities, scoliosis in particular, represent the most prevalent type of orthopedic deformities in children and adolescents, while idiopathic scoliosis (IS) represents the most common form of scoliosis. By definition, idiopathic scoliosis is a three-dimensional spinal curvature of at least $10^{\circ}$ (Cobb angle) in the coronal plane that develops during growth in otherwise healthy children. Adolescent IS (AIS), generally defined by a clinical diagnosis made in children between the ages of 8 and 18 years [1], affects an average of $4 \%$ of the pediatric population globally, and approximately 7 million people in the USA. One out of every six children diagnosed with IS will have a progressive curve that requires active treatment, and there is a predominance of females among the severe cases. In 2010, the estimated total hospital costs in USA for all patients released from the hospital with an AIS diagnosis, excluding professional fees and non-covered charges, was US $\$ 2.7$ billion. Patients today are treated in a substantially similar manner to those 20 or 30 years ago: observation (mild deformities: between $10^{\circ}$ to $25^{\circ} \mathrm{Cobb}$ angle); bracing (moderate curves: $25^{\circ}$ to $35^{\circ}$ Cobb angle); and spinal surgery (severe, progressive curves: $\geq 45^{\circ} \mathrm{Cobb}$ angle) as last resort. Unfortunately, there is no proven method or test available to identify children or adolescents at risk of developing AIS, or to identify which of the affected individuals may require treatment due to the risk of progression.
Consequently, the application of current treatments, such as bracing or surgical correction, is delayed until a significant deformity is detected or until a significant progression is clearly demonstrated, resulting in a delayed and less than optimal treatment [2].

Because there has been little progress in understanding the etiology or progression of AIS, the approach to treatment has remained unchanged. Early detection of scoliosis could lead to successful and less invasive clinical outcomes by broadening the range of treatment options and increasing their effectiveness. Today, there is one genetic test that is currently available. IS is diagnosed through physical examination and, after detection, genetic tests such as ScoliScore ${ }^{\mathrm{TM}}$ (Transgenomic, Inc., NE, USA) estimate the probability of disease progression based on genomic markers and clinical indices. However, such a test does not alter treatment options available. It is likely that a curvature that is detectable through physical examination, generally in children at approximately age 12 years, occurs after the biochemical cascade of the etiology has taken root and progressed sufficiently to cause physical deformity. It is our opinion that detecting scoliosis at an earlier stage, with a better understanding of the biology of the deformity, will allow intervention when the biochemical processes that cause curvature of the spine can be mitigated or reversed.

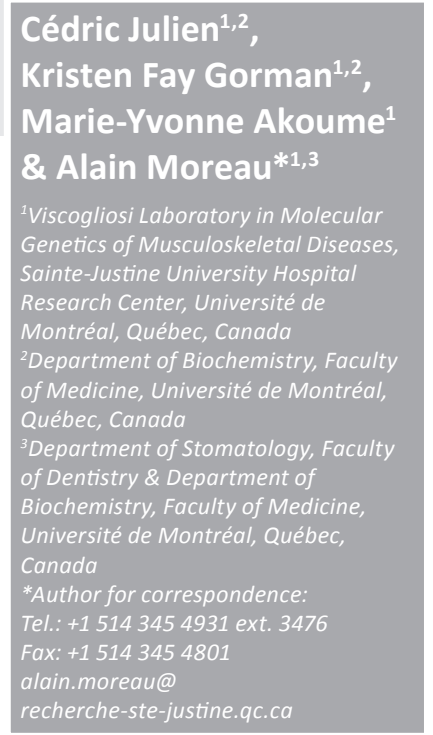

Future
Medicine $\mathrm{fS}$ 
Although a genetic basis is widely accepted for AIS, multiple genes and loci have been associated and none are confirmed [3]. Thus, genetic and phenotypic heterogeneity clearly increase the difficulty of studying complex diseases such as AIS. Although one could argue that personalized medicine will benefit from the impressive improvements in genetic/molecular mapping and development of annotation and analytic tools, we have to give meaning to such data. Here we describe a combined functional, biochemical and genetic screen to understand the myriad of factors influencing the predisposition and pathogenesis of AIS. This integrated approach to understanding complex diseases such as AIS will allow for better diagnoses and prognoses, as well as the development of personalized therapies.

\section{Genetic studies \& their clinical utility}

AIS is typical of a complex disease in that it is explained by the association of genetically predisposing factors interacting with environmental factors and/or disease modifiers. Moreover, clinical manifestations could be very different from one individual to another (i.e., onset, morphology, severity, progression and response to standard treatments), and this may be due to biomechanical and behavioral considerations confounding the primary etiology. The relatively high frequency of AIS (3-4\%) in most (if not all) populations and its strong genetic underpinnings (15\% of AIS cases occur in families with several affected members, while the risk of developing a scoliosis increases up to $40 \%$ when one of the parents is affected) relative to other well-studied complex diseases predicts good power for genomewide association studies (GWAS). However, with complex genetic diseases, phenotypic and genetic heterogeneity can effectively dilute positive associations. Indeed, the Intermountain Region of the USA has reported a familial incidence as high as $97 \%$ for probands, and yet no causative genes have been identified [4]. Although, generally, an increase in the number of individuals would contribute to improve a study's power to detect a genetic effect, with complex diseases such as AIS, heterogeneity is a major obstacle that cannot be overcome by such means because there is likely to be population-specific genetic associations. This may explain why so few positive associations for AIS have been replicated in different populations. With regard to genetic testing, this limitation becomes a serious consideration. For example, the ScoliScore ${ }^{\mathrm{TM}}$ multigene test only provides a score associated with the likelihood of curve progression for Caucasian American or Canadian patients between $9-13$ years old who have already been diagnosed with AIS [5-7]. The clinical validity of a genetic test defines how well the results detect or predict a disorder (scoliosis) or final outcomes of interest (magnitude of spinal deformity progression and/or risk of developing a specific comorbidity). Unfortunately, this test and associated markers have never been replicated anywhere else in the world, and do not have a positive predictive value about the risk of developing a severe scoliosis. Thus, although in concept the identification of genetic variants correlating to disease predisposition and/or progression would be on asset for clinicians, the complexity of the disease and an apparent population-based genetic heterogeneity can lead to limited successes.

\section{Characterization of biological endophenotypes associated with AIS}

To address the phenotypic and genotypic heterogeneity of AIS, we identified a common disease mechanism among all patients and then subdivided these patients based on their biochemistry. These biological endophenotypes, defined by Moreau et al., can then partition biological variation and thus increase the power to detect genetic associations [8-11]. By definition, an endophenotype is a term from genetic epidemiology used to parse behavioral symptoms into more stable phenotypes with a clear genetic connection [101]. This notion was first suggested by Bernard John and Kenneth R Lewis in an article called "Chromosome variability and geographic distribution in insects", where a specific geographic distribution of insects is more likely due to internal endophenotypes instead of obvious external phenotypes [12]. The concept of endophenotypes has been widely applied to complex psychiatric disorders such as schizophrenia. Endophenotypes are believed to have a polygenic background with multifactorial origins. They are associated with illness in the population, are heritable and can manifest in an individual whether disease is active or not, and endophenotypes segregate with illness within families $[13,14]$. Moreover, endophenotypes are usually present in affected and nonaffected family members at a higher rate than in the general population [15].

In complex genetic disorders such as AIS, endophenotypes have potential utility both in identifying risk genes and in illuminating the pathophysiology. We have identified three distinct heritable endophenotypes for AIS, based on the previously reported $\mathrm{G}$ inhibitory $(\mathrm{Gi})$ signaling dysfunction in AIS patients [11]. Using multiple cell types, a whole-cell assay was developed using 
cellular dielectric spectroscopy to measure the activity of G-protein-coupled receptors among AIS patients, controls and asymptomatic children with a family history of AIS. These observations led us to classify patients into three distinct groups (referred to as FG1, FG2 and FG3), depending on the maximal Gi response observed after stimulation. This disparity is very specific, as such patterns are not observable among healthy controls. The use of these endophenotypes has allowed for in-house patient stratification that has been correlated to explicit molecular expression profiles and differential prognoses [YUAN Q ETAL. GENOME-WIDE EXPRESSION PROFILING OF IDIOPATHIC SCOLIOSIS ENDOPHENOTYPES (2012), MANUSCRIPT IN PREPARATION]. The use of endophenotypes has allowed us to highlight some specific clinical features of the disease. Importantly, the occurrence of severe scoliosis cases (Cobb angle $\geq 45^{\circ}$ ) is strikingly different among each biological endophenotype. In our French-Canadian pediatric cohort, the percentages of severe cases are $13 \%$ for FG1, $60 \%$ for FG2 and 27\% for FG3 endophenotype. These findings are currently being validated in two independent cohorts. Of note, our blood tests should be viewed first as prognosis tools in affected patients to establish at an early stage the risk of disease progression. However, the same tests applied to asymptomatic children at risk (e.g., offspring of scoliotic parents) could be used to predict the risk of developing scoliosis before disease onset.

\section{Genetic studies: our reverse engineering approach using AIS endophenotypes}

Our research has demonstrated different molecular profiles for the distinct endophenotypes for AIS, and these should, in concept, have genetic distinctions. Importantly, the use of endophenotypes in complex genetic studies has the advantage of adding power for gene detection. We have recently reviewed all the important genetic studies published to date in the field of AIS [3]. Like most complex diseases, few studies have been successfully replicated, which may be due to the lack of agreement about the selected disease phenotypes, the natural variability associated with ethnicity and simply the lack of attempts for candidate gene studies. The overall picture remains the same: AIS has a heterogeneous predisposing genetic etiology.

For complex heritable diseases there are two major competing hypotheses regarding the genetic architecture: the disease is caused by multiple common variants of minor effect segregating in the population, or by a few rare variants of major effect [16-18]. The latter hypothesis has been advocated more recently in light of the minor effect correlations from GWAS [19,20]. To identify the genes impacted or directly involved in each AIS endophenotype, we used a multiplexed genetic approach: exome sequencing, GWAS, individual sequencing of candidates and gene-expression analyses. One strategy is to identify genetic variation that will identify at-risk individuals prior to the onset of curvature, or to identify individuals at risk for curve progression. For AIS, if genetic correlates occur at a minor frequency in the population, then we expect that whole-exome sequencing of many patients and controls will reveal these rare variants that would not be detected by standard GWAS. However, a possible pitfall of such an approach is that the exome accounts for only $1.5 \%$ of the total genome [102], and it is possible that AIS is caused by variants localized in noncoding regions too. Indeed, recent analysis for the ENCODE project has demonstrated that prior results from GWAS have disease associations enriched in those regulatory regions [103]. Thus, we have adopted a complementary approach of exome sequencing combined with GWAS to elucidate important gene and/or regulatory correlates for the disease. Common single nucleotide variants (SNVs) often lead to conflicting results between studies. Heterogeneity between populations and different degrees of admixture are responsible for this disparity. An important strength for our GWAS is that our initial population consists of only FrenchCanadians (Great Montreal area, Province of Quebec). Our preliminary analyses confirm population homogeneity. Indeed, Quebec has a higher incidence of AIS compared with other countries. Confirmation of genetic correlates will include targeted resequencing among families, and also validation in independent cohorts outside of Quebec. We may consider common variants as tags that are in strong disequilibrium with disease-causing SNVs. Targeted resequencing of genomic regions defined by such tags should be carried out to reinforce the potential involvement of an identified locus. In the end, we may be able to connect population-specific SNVs together.

The nature of genetic risk factors is varied. Approaches described above allow us to identify several SNVs and insertions/deletions that can affect the protein sequence and structure (missense and nonsense). Certain silent variations can affect pre-mRNA splicing [21]. SNVs 
located in noncoding regions could be responsible for variability in gene expression, while copy number variations can have a wide spectrum of effect on protein levels, function and integrity. Because we have identified differential prognoses correlated with each AIS endophenotype, a second priority for genetic studies is to identify genetic markers that segregate differently among those biological endophenotypes. Therefore, all of our genomic analyses are stratified according to our functional classification or endophenotypes. Furthermore, to identify the pathways that are differentially impacted, we used individual microarrays and quantitative PCR on AIS patients. These results will validate important genes involved in the distinctiveness of each AIS endophenotype and act to bridge the genetics and our biochemical datasets. Moreover, knowledge generated from our experiments can guide us in the genetic dissection of the affection, and help us in our effort to correlate potential candidate $S N V s$ located within regulatory regions with specific gene-expression patterns.

\section{Conclusion}

Although AIS has a genetic basis, previous studies have not been able to elucidate its etiopathogenesis. We are using a comprehensive approach to identify genetic regions that are associated with AIS. A strength of our approach is that we have previously identified robust biochemical endophenotypes, and thus have a biological context that can guide our biostatistical interpretation of data. In addition to this, we use a nonhypothesis-driven approach to discover new disease correlates in the genome. The search for genetic factors specifically associated with each of our described endophenotypes is promising for the improvement of diagnostic and prognostic innovations. We strongly believe that whole-exome sequencing followed by targeted resequencing will help us to discover new variants linked to AIS. Indeed, we have recently identified more than 300 new SNVs from a study using 70 AIS patients (21-26 individuals per group) and 25 matched controls. The majority of the SNVs are uniquely distributed among our functional endophenotypes. Candidate genes thus identified will be directly sequenced in extended cohorts for validation and replication. While exome analysis has the potential to reveal rare disease variants, GWAS have the potential to reveal important regulatory elements. In combination with our gene-expression experiments we can elucidate important biological pathways specific to the differential AIS prognoses (i.e., endophenotypes). Thus, by highlighting the pathways involved and connecting them to the Gi-protein signaling defect described so far, we will be in a position to understand, at least in part, the molecular and cellular aspects of the physiopathology of AIS.

\section{Future perspective}

We agree that the clinical utility of a test is the likelihood that using the test to guide management will significantly improve health-related outcomes. Idiopathic scoliosis is common enough that a direct study that begins with biochemical, functional or genetic testing and follows through to clinical outcomes should be possible in a multicenter protocol. In this clinical scenario, early detection of progressive scoliosis is not only critical to successful and less-invasive clinical outcomes, but broadens the range of treatment options for clinicians and increases effectiveness through personalized medicine (Figure 1). Evaluation of the clinical outcomes of AIS patients according to their biological endophenotypes reveals in two distinct cohorts (Montreal, Canada and Milan, Italy) that those classified with FG2 endophenotype are more susceptible to developing severe scoliosis, while those with FG1 endophenotype present a much lower risk of disease progression. Indeed, approximately $60 \%$ of FG2 AIS patients required surgery, compared with only $5-13 \%$ of those classified in FG1 [Акочме M-Y ET $A L$. Gi SIGNALING DifFERENTIAL DisRUPtion OCCURS IN ADOLESCENT IDIOPATHIC SCOLIOSIS (2012), MaNUSCRIPT IN preparation]. Our tests are currently in clinical validation in Montreal and Milan in scoliotc patients at an early stage, as well as in asymptomatic population at risk (offspring of scoliotic parents and individuals with familial antecedents).

The ability of the diagnostic/prognostic assays to better confirm the scoliosis condition of the patient, particularly in terms of prognosis, will allow for earlier and more appropriate interventions. It is anticipated that primary care physicians (e.g., pediatricians) will play a more active role in scoliosis monitoring and treatment, which will eventually lead to a reduction in hospital visits and costly interventions. Improving patient stratification and disease staging, in combination with new clinical decision-making tools and algorithms, should allow spine surgeons to reduce their surgical waiting list by selecting first patients at high risk of disease progression before the spinal deformity is too advanced. The rationale of this strategy stems from the fact that 


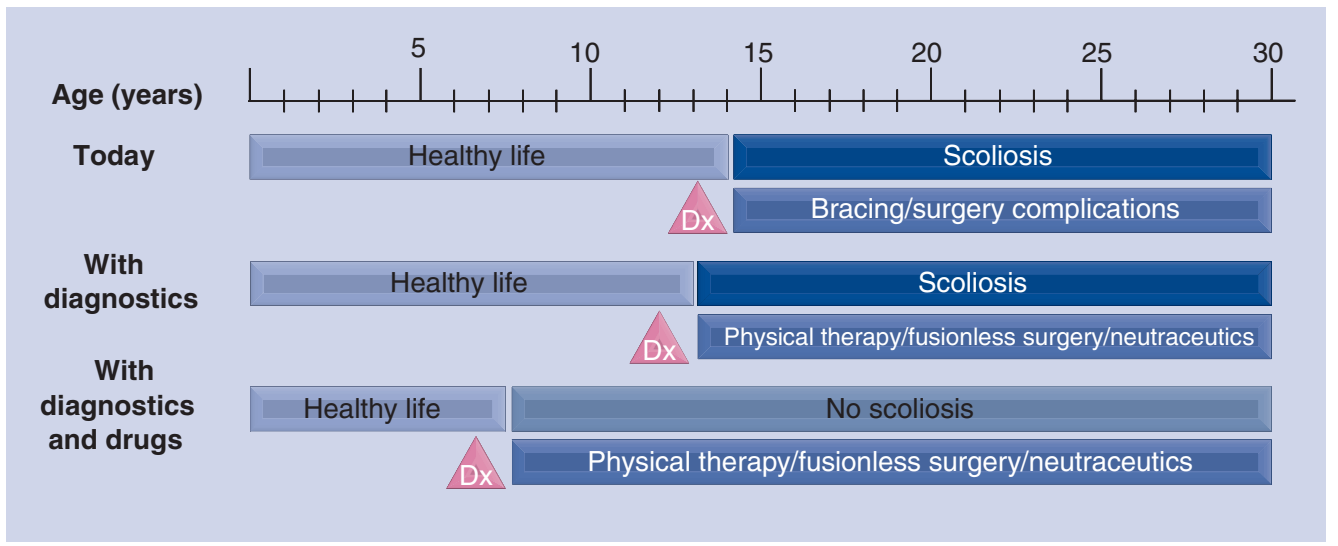

Figure 1. New continuum of pediatric healthcare in pediatric scoliosis. Pediatric scoliosis is a chronic health condition that will impact individuals throughout life. Therefore, early detection broadens the range of treatment options and increases effectiveness when the biochemical processes that cause spinal curvature can be halted or reversed. Development of comprehensive predictive tests could not only help to predict disease, but could have a significant impact on treatment and clinical decisions by predicting particular clinical outcomes leading to cost-effective personalized health solutions.

Dx: Diagnostic.

less deformed and immature spines are easier to correct because they are more flexible, as opposed to older patients with more deformed and rigid spines leading to less optimized corrections, more complications and increased health costs. Therefore, we expect that a better patient stratification/ selection will also translate into a reduction in operating room time and instrumentation costs (fewer levels having to be instrumented), less complications (decreased blood loss and infection rates) and faster patient health recovery. Improving patient's stratification by means of genomic predictive tests would prevent serious psychological side effects and reduce the burden of scoliosis by avoiding unnecessary invasive treatments (e.g., bracing) in patients who will not respond

\section{Executive summary}

\section{Current situation}

- Idiopathic scoliosis is the most common orthopedic condition requiring surgery in adolescents, and affects $4 \%$ of this population.

- Most patients requiring treatment are females (90\%).

- Today, the global diagnostic and therapeutic market for adolescent idiopathic scoliosis (AIS) is well over US\$2.5 billion dollars annually in North America.

- Curvature detectable through physical examination is generally identified in children at approximately age 12 years, after the biochemical cascade has taken root and progressed sufficiently to cause physical deformity.

- Currently, there are no US FDA-cleared 'presymptomatic' diagnostic tests available for assessing scoliosis in pediatric or adolescent patients. While there may exist products/diagnostics that claim to aid in identifying scoliosis precursors, most of the laboratory-developed tests are not governed by the FDA. They are regulated through the Clinical Laboratory Improvement Amendments of the Department of Health and Human Services and the College of American Pathologists. Clinical Laboratory Improvement Amendments certification and a College of American Pathologists number are required before it can be used clinically. Both are rigorous processes. Generally, if the test is one that leads to a serious intervention, such as surgery, the FDA is involved; however, most molecular diagnostics are not.

- The standard of care for scoliosis has not changed in any significant manner in decades. Patients today are treated in a substantially similar manner to those 20 or 30 years ago - observation, bracing and fusion as a last resort.

\section{Future perspective}

- Endophenotypes will help in defining the genetic background, thus leading to a better understanding of the AIS physiopathology.

- Evaluation of the clinical outcomes of AIS patients according to their biological endophenotypes reveals those that are more susceptible to developing severe scoliosis, while others are at a much lower risk of disease progression. Indeed, approximately $60 \%$ of AIS patients with the FG2 endophenotype require surgery, compared with only $13 \%$ of those classified with FG1.

- The most immediate application of validated diagnostic/prognostic tools is to help spine surgeons to identify high-risk disease progressors who could benefit from surgery at an early stage when the deformity is not too advanced to maximize the correction, to reduce the number of levels instrumented, the recovery time, complication rate and costs.

- Improving AIS patients' stratification will facilitate the development of personalized care and the first pharmacological treatments in the field of orthopedics. 
to those treatments due to their genetic predisposition, while too often braces are prescribed to children that are nonprogressors. Furthermore, prevention of scoliosis could be possible in the near future through tailored pharmacological therapies, which requires the development and validation of presymptomatic tests to identify children at risk of developing a scoliosis.

We acknowledge that the impact on clinical practice is speculative at this point and will require cost-effectiveness analyses to allow a precise estimate of the cost impact of the current practice, but also to what extent these costs will be modified by the adoption of the diagnostic/prognostic assays in clinical practice. Besides estimating the cost-effectiveness of the diagnostic/prognostic assays with the cost-utility analysis, a budget impact analysis model should also be developed. The cost-utility analysis will inform the decision makers on the efficiency of the assays; on the other hand, the budget impact analysis will provide an estimate of the overall economic impact of adopting the diagnostic/prognostic assays.

\section{Financial \& competing interests disclosure}

This work was supported in part by research grants from The Yves Cotrel Foundation at Institut de France (to A Moreau), The Canadian Institutes of Health Research (CIHR PP2 - 99466 to A Moreau), Fourth Dimension Spine LLC (USA) and Génome Québec (to A Moreau). KF Gorman is the recipient of an Excellence Postdoctoral Scholarship from CHU Sainte-Justine Foundation and MENTOR training program/CIHR. The authors have no other relevant affiliations or financial involvement with any organization or entity with a financial interest in or financial conflict with the subject matter or materials discussed in the manuscript apart from those disclosed.

No writing assistance was utilized in the production of this manuscript.

\section{References}

Papers of special note have been highlighted as: - of interest

1 Miller NH. Adolescent idiopathic scoliosis: etiology. In: The Pediatric Spine. Weinstein SL (Ed.). Lippincott, Williams \& Wilkins, Philadelphia, PA, USA, 347-354 (2001).

2 Ali Fazal M, Edgar M. Detection of adolescent idiopathic scoliosis. Acta Orthop. Belg. 72(2), 184-186 (2006).

3 Gorman KF, Julien C, Moreau A. The genetic epidemiology of idiopathic scoliosis. Eur. Spine J. 10, 1905-1919 (2012).

4 Ogilvie JM, Braun J, Argyle V et al. The search for idiopathic scoliosis genes. Spine 31(6), 679-681 (2006).

5 Ward K, Ogilvie JW, Singleton MV et al. Validation of DNA-based prognostic testing to predict spinal curve progression in adolescent idiopathic scoliosis. Spine 35(25), e1455-e1464 (2010)

6 Ogilvie JW. Update on prognostic genetic testing in adolescent idiopathic scoliosis (AIS). J. Pediatr. Orthop. 31 (Suppl. 1), S46-S48 (2011).

7 Carlson B. ScoliScore AIS prognostic test personalizes treatment for children with spinal curve. Biotechnol. Healthc. 8(2), 30-31 (2011).

8 Moreau A, Wang DS, Forget $S$ et al. Melatonin signaling dysfunction in adolescent idiopathic scoliosis. Spine 29(16), 1772-1781 (2004).

- This seminal paper presents the first experimental evidence of a differential impairment of Gi-signaling in adolescent idiopathic scoliosis (AIS) patients. The authors used bone-forming cells (osteoblasts) derived from intraoperative bone specimens taken from AIS patients to assess melatonin receptor signaling activity using a cAMP whole-cell assay.

9 Azeddine B, Letellier K, Wang DS et al. Molecular determinants of melatonin signaling dysfunction in adolescent idiopathic scoliosis. Clin. Orthop. Relat. Res. 462, 45-52 (2007).

- Provides further results showing the molecular basis of the three functional groups associated with AIS patients.

10 Moreau A, Akoume MY, Azeddine B et al. Molecular and genetic aspects of idiopathic scoliosis. Blood test for idiopathic scoliosis. Orthopade. 38(2), 114-121 (2009).

11 Akoume MY, Azeddine B, Turgeon I et al. Cell-based screening test for idiopathic scoliosis using cellular dielectric spectroscopy. Spine 35(13), 601-608 (2010).

- Further confirms the results obtained previously with cAMP assays using the cellular dielectric spectroscopy method, a label-free system. Both methods allow the stratification of AIS patients in the same three functional groups or biological endophenotypes.

12 John B, Lewis KR. Chromosome variability and geographic distribution in insects. Science 152(3723), 711-721 (1966).

13 Gershon ES, Goldin LR. Clinical methods in psychiatric genetics, I. Robustness of genetic marker investigative strategies. Acta Psychiatr. Scand. 74(2), 113-118 (1986).
14 Gottesman II, Gould TD. The endophenotype concept in psychiatry: etymology and strategy intentions. Am. J. Psychiatry. 160(4), 636-645 (2003).

- Concept and potential use of endophenotypes in psychiatric disorders. According to the authors, endophenotypes allow a greater potential for detecting candidate genes or genomic regions than the syndrome itself. For a greater gain in usefulness, endophenotypes should meet certain criteria such as heritability, primarily state independence or association/ cosegregation with the studied disease in populations/families. Moreover, endophenotypes can be used as biological signatures for disease classification and diagnosis.

15 Leboyer M, Bellivier F, Nosten-Bertrand M, Jouvent R, Pauls D, Mallet J. Psychiatric genetics: search for phenotypes. Trends Neurosci. 21(3), 102-105 (1998).

16 Bodmer W, Bonilla C. Common and rare variants in multifactorial susceptibility to common diseases. Nat. Genet. 40(6), 695-701 (2008).

- The authors discuss the genetic contribution of common and rare variants to the etiology of chronic diseases. The identification of common variants requires large studies to achieve statistical significance. However, their effects appear to be elusive as they become lost in the context of a fluctuating environmental background. Rare variants, in association with common variants, may 
contribute to multifactorial inheritance and may be useful for understanding the underlying etiopathology of chronic complex diseases.

17 Manolio TA, Collins FS, Cox NJ et al. Finding the missing heritability of complex diseases. Nature 461(7265), 747-753 (2009).

18 Li B, Leal SM. Methods for detecting associations with rare variants for common diseases: application to analysis of sequence data. Am. J. Hum. Genet. 83(3), 311-321 (2008).
19 Neale BM, Rivas MA, Voight BF et al. Testing for an unusual distribution of rare variant. PLOS Genet. 7(3), e1001322 (2011).

20 Myers RA, Casals F, Gauthier J et al. A population genetic approach to mapping neurological disorder genes using deep resequencing. PLOS Genet. 7(2), e1001318 (2011).

21 Cartegni L, Chew SL, Krainer AR. Listening to silence and understanding nonsense: exonic mutations that affect splicing. Nat. Rev. Genet. 3(4), 285-298 (2002).

\section{Websites}

101 Wikipedia, the free encyclopedia: endophenotype.

http://en.wikipedia.org/wiki/Endophenotype

102 The encyclopedia of DNA elements (ENCODE).

http://genome.ucsc.edu/ENCODE

103 Nature ENCODE.

www.nature.com/encode/\#/threads 